\title{
A New Approach for Enhanced Communication to LEO Satellites
}

\author{
E. Gill ${ }^{1}$, Ch. Verhoeven ${ }^{2}$, \\ Delft University of Technology, Kluyverweg 1, 2629 HS Delft, The Netherlands \\ K. Gill ${ }^{3}$ \\ Universität Konstanz, Universitätsstrasse 10, 78457 Konstanz, Germany \\ and \\ M. de Milliano ${ }^{4}$ \\ Science \& Technology B.V., Olof Palmestraat 18, 2616 LR Delft, The Netherlands
}

\begin{abstract}
Operations of satellites in Low-Earth Orbit (LEO) have been based on exploiting the maximum time of visibility of satellites over their ground stations. In this paper, it is shown that a key parameter for communication between a satellite and a ground station is not the time of visibility but the amount of telemetry data which can be transmitted from the satellite to the ground station in the downlink. Given the fact that advanced satellites may allow a variety of data rates for downlink, switching on and off the transponder at higher elevations (i.e. at lower slant ranges) than the minimum possible elevation allows for a higher data rate, and thus, despite of less contact time, for a higher total data volume to be transmitted during a given pass. The paper motivates this new approach for space-ground communication of satellites in LEO on the background of recent technology advances and develops the theoretical framework for enhanced communication. To this extent, an analytical relation is established of the total data volume which can be downlinked for a single pass as a function of the elevation at which the transponder shall be switched on and off. The analysis is limited to a circular LEO and zenith passes. Numerical simulations of the total data volume as a function of this minimum elevation angle are presented as well. Finally, associated opportunities and challenges for enhanced communications are identified and discussed. Conclusions are drawn for future optimized operations of LEO satellites based on given ground segments.
\end{abstract}

\section{Nomenclature}

$=$ Data volume

$=$ Energy

$=$ Elevation

$=$ Gravitational constant

$=$ Spacecraft altitude

$=$ Boltzmann constant

$=$ Loss factor

$=$ Margin factor

$=$ Earth mass

$=$ Noise

\footnotetext{
${ }^{1}$ Professor and Chairholder Space Systems Engineering, Faculty of Aerospace Engineering, AIAA member.

${ }^{2}$ Associate Professor, Faculty of Electrical Engineering, Mathematics and Computer Sciences.

${ }^{3}$ Student, Fachbereich Physik.

${ }^{4}$ Staff Member.
} 


\section{Introduction}

core element in the process of designing the communication subsystem of satellites is the link budget. A A key parameter in this budget is the applied minimum elevation angle. Typically, this value is applied as input parameter in the process which primarily depends on ground equipment, radio-frequency regulations, operation strategies and horizon masks ${ }^{1}$. Minimizing this value for ground station operations of LEO satellites allows a maximum time of visibility ${ }^{2}$. Similar considerations are applied from a satellite operator point of view where the application of minimum elevation angles as low as possible is the preferred approach ${ }^{3}$.

Although extensive literature exists on the optimization of satellite operations with respect to increased data throughput, an analysis of transponder scheduling in relation to total data volume and elevation has, to our knowledge, not yet been performed. In Ref. 4, an optimization of network planning and satellite operations has been done in a general context of mission priority and science return for deep space networks. Ref. 5 analyses timevarying satellite links which have flexibility in information bit rate and/or transmit power for improved throughput. The elevation plays an important role in specific problems such as visibility conditions and rain attenuation. Two examples of literature in these fields are Ref. 6 and 7, which discuss the relevance of elevation for low-gain nontracking antennas and models for rain attenuation involving elevation, respectively.

Instead, in this work a new approach is investigated which derives the minimum elevation angle from the maximum of the total data volume which can be transferred during a pass ${ }^{8}$. Considering an adaptable data rate,, switching on a transponder at a higher minimum elevation angle (i.e. at lower slant ranges, i.e. later in the pass) and turning the transponder off at this same higher minimum elevation angle later in the pass (i.e. at lower slant ranges, i.e. earlier with respect to the end of the pass) allows a higher data rate which still satisfies the link budget. Thus, a trade-off may be performed between time of communication and data rate to maximize the total achievable data volume.

An analytical analysis of the pass geometry of satellites in a circular LEO has been performed which shows that the minimum elevation angle at which the per-pass data volume is maximum depends only on three parameters: the Earth's radius, the satellite altitude, and the maximum elevation angle. Numerical simulations have been performed for various scenarios. We limit our analysis to zenith passages, where the minimum elevation angle at which the total per-pass data volume is maximized, is about 42 degrees at a satellite altitude of $600 \mathrm{~km}$.

Technology advances allow for various data rates to be selected, and, in the future, as long as the bit energy to noise power density is sufficient, flexible data rates would enable optimized communications under varying channel conditions and available power. However, this will increase the complexity of the system both on ground and in space and poses requirements on the applied protocol.

The presented approach to enhanced space-ground communication for LEO satellites opens possibilities for reorganizing ground operations such that freed ground station time can be used for other missions and thus enables maximum use and optimum exploitation of sparse and costly ground segments in the future.

The paper is organized as follows: Technology aspects of advanced spacecraft communications are presented in Chapter II. The link analysis is performed in Chapter III. Chapter IV addresses ground station operations context and needs and discusses opportunities and challenges of the presented approach. A summary and conclusions are provided in Chapter V.

\section{Technology}

New technologies offer an abundance of possibilities to create systems with a performance that could not even be imagined a few years ago. Especially in communication technology the impact is huge. Due to the massive use of commercial wireless communication systems the electromagnetic spectrum has become crowded and inefficient use of frequency bands cannot be tolerated. Modern radio design focuses on optimal use of the spectrum. This leads to complex scenarios and designs, but fortunately modern technology offers all ingredients to implement these designs in a reliable and commercially interesting way. Radio circuits designed in the main-stream CMOS technologies can 
be easily accompanied by enough digital processing power to optimize the performance of the analog circuits ${ }^{9}$. In addition, functions formerly performed by an analog circuit are shifted to the digital domain ${ }^{10}$. The immediate benefit of this digitalization is the increased flexibility. It has become much easier to design reconfigurable radios that can be adapted or adapt themselves to changing requirements in real-time $\mathrm{e}^{10-13}$.

It has been shown that it is possible to design in CMOS efficient radio systems that are able to optimally use the available radio link ${ }^{14}$ and also that radios in CMOS technology have a future application in space ${ }^{15-17}$. Especially in the range of very small satellites, such as pico-, nano- ${ }^{18}$, or micro-satellites built by smaller consortia for commercial services, the number of satellites in operations will rapidly grow. The frequency bands for satellite communication will become more and more crowded. However, similar radio systems designs in similar technologies as used in terrestrial applications can become part of the solution.

One of the parameters to be optimized in, e.g., the Global System for Mobile Communications (GSM) is the duration of radio communication. Both the radio circuit and the coding are optimized in such a way that the time needed for communication with the base station is short as possible, leaving more time for the base station to serve other phones. A similar situation is found for satellite communication. The number of ground stations and frequency bands is limited, so the time used for communication should be as short as possible and the radio systems should be optimized for that. In commercial mobile radio systems, the circuit designs and the system concepts are such that the effort to optimize the system for a scenario with a different number of base stations or mobile units is technology wise not very complicated anymore. Flexibility, re-configurability and adaptability are key-words for modern microelectronic systems. However, this must be technically feasible now or in the near future and account for systems already in operation ${ }^{19}$. A first step forward would be the design of a general radio system (chip) which can be adapted or programmed to optimally perform in a specific satellite mission in near real-time. When the orbit parameters and communication needs are known, an optimal radio system can be implemented without the need for a dedicated technology or a dedicated and thus expensive and time-consuming Integrated Circuit (IC) design effort. Instead, the radio set-up can be uploaded to the satellite, or, alternatively, be available on-board as a set of predefined operating modes to be selected as soon the communication needs of a pass become known. From this it follows that the implementation of a radio to support a flexible data rate, stepwise or even continuously, is feasible and, as will be shown in the sequel, very promising.

\section{Link Analysis}

The total telemetry data volume $D$ to be received during a single ground station pass in between the transmission begin and end times $t_{\mathrm{b}}$ and $t_{\mathrm{e}}$ is given as

$D=\int_{\mathrm{t}_{\mathrm{b}}}^{\mathrm{t}_{\mathrm{e}}} R(t) \mathrm{d} t=R_{\max }\left(t_{e}-t_{b}\right)$,

where $R$ is the data rate. Note that in Eq. (1), we assume for the sake of simplicity that a maximum possible data rate $R_{\max }$ is selected which satisfies the link budget, but which is kept constant over the transmission time. Thus, the data volume depends on two key aspects: the data rate which is a function of the technology applied and the slant range between ground station and satellite and the transmission time $t_{\mathrm{t}}=t_{\mathrm{e}}-t_{\mathrm{b}}$ which is a function of the visibility and pass geometry. Operations of LEO satellites typically apply a constant data rate over the transmission times during a single pass.

The data rate $R$ is a key factor in the link budget which is described in the simplified equation ${ }^{1}$

$$
\frac{E_{b}}{N_{0}}=\frac{P L_{t} G_{t} L_{p} L_{a} G_{r} L_{r} M}{k T_{s} R} \text {, or } R=R_{C} L_{P}
$$

where $E_{\mathrm{b}}$ is the received bit energy, $N_{0}$ is the noise density, $P$ is the transmitted power, and $L_{\mathrm{t}}$ and $L_{\mathrm{r}}$ as well as $G_{\mathrm{t}}$ and $G_{\mathrm{r}}$ are the losses and gains of the transmitter and receiver chains, respectively. Further, $L_{\mathrm{p}}$ and $L_{\mathrm{a}}$ is the path loss and atmospheric loss, $M$ is the minimum link margin, $k$ is the Boltzmann constant and $T_{\mathrm{s}}$ is the system temperature. In the right-side of Eq. (2), $R_{\mathrm{C}}$ comprises terms implicitly defined by the left side equation. In Eq. (2), the path loss is modeled as ${ }^{1}$

$$
L_{p}=\left(\frac{\lambda}{4 \pi \rho}\right)^{2},
$$

where $\lambda$ is the wavelength of the signal and $\rho$ the range, or slant range, between the ground station and the satellite. Finally, the range may be expressed as a function of the elevation $E$ of the satellite above the ground station's horizon according to ${ }^{2}$ 


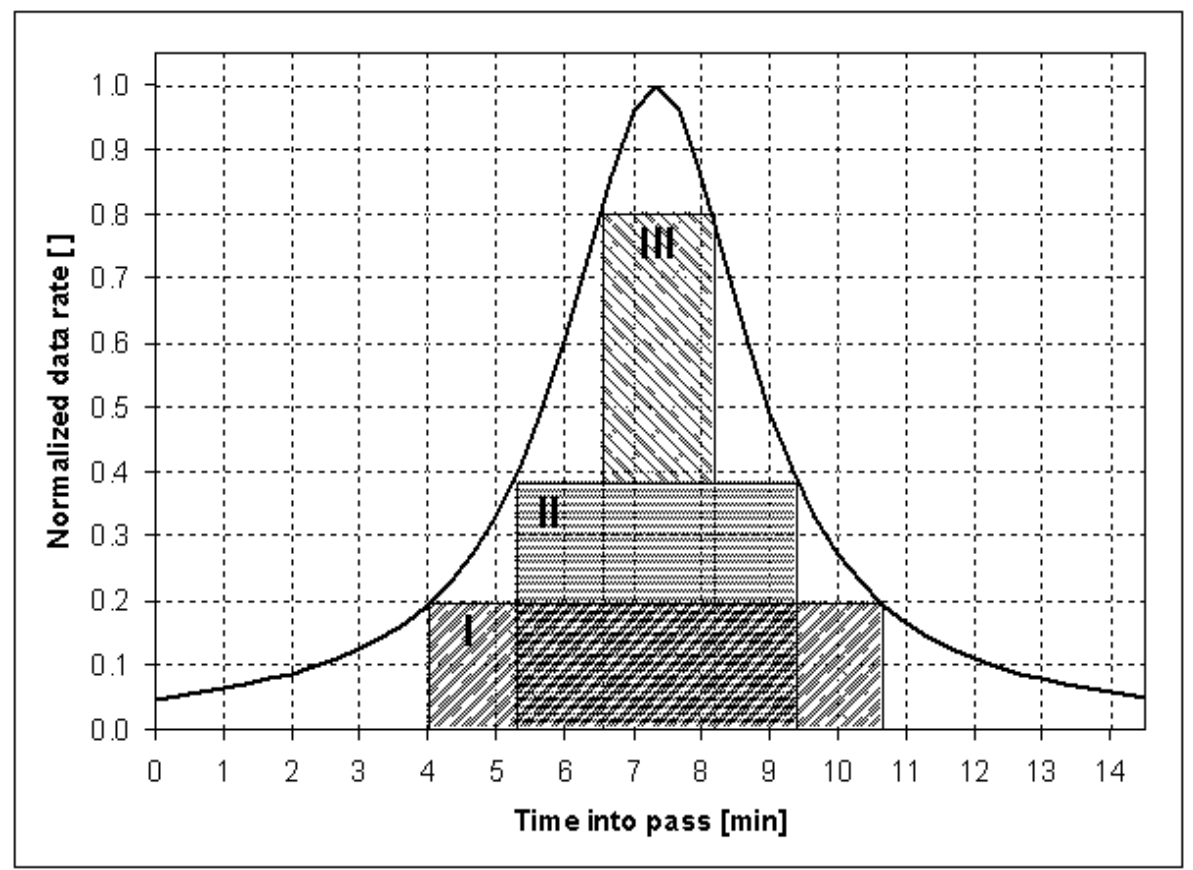

Figure 1 Normalized data rate as a function of the time into pass for a sample zenith passage of a satellite in a circular orbit at $700 \mathbf{~ k m}$ altitude. The total data volume is the area of the rectangles indicated in the figure. Three scenarios are indicated with different transmission times and associated different data rates. Scenarios I and III allow the same total data volume. Scenario II is superior to scenarios I and III.

$$
\rho=R_{\oplus}\left(\sqrt{\frac{r^{2}}{R_{\oplus}^{2}}-\cos ^{2} E}-\sin E\right),
$$

where $R_{\oplus}$ is the Earth's equatorial radius and $r$ is the satellite's geocentric distance. With these models, a data rate which can technologically be selected among various values and a given link budget, we may regard (2) with all factors kept constant except the path loss and the data rate as a function of the range which is again a function of the elevation. A decrease in range will reduce the path loss and, with all other factors in the link budget kept constant, will allow a higher data rate.

The second factor which determines the total data volume in Eq. (1) is the transmission time. In the following we limit our considerations for the sake of simplicity to zenith passes over the ground station. The general conclusions are valid for non-zenith passes as well. For zenith passes, the transmission time is given as ${ }^{8}$

$$
t_{t}=\frac{r^{3 / 2}}{\sqrt{G M_{\oplus}}}\left(\pi-2 E-2 \arcsin \left(\frac{R_{\oplus}}{r} \cos E\right)\right) .
$$

The maximum normalized data rate possible during a single ground station pass as a function of the time into the pass is illustrated in Fig. 1. The figure exhibits the relation by way of example of a zenith pass of a satellite in a circular orbit at $700 \mathrm{~km}$ orbital altitude. The total area under the curve in Fig. 1 is the maximum theoretical total data volume achievable in a single pass would the data rate continuously change depending on the path loss. However, as discussed above, once the transmission has started, the data rate is kept constant over the transmission interval in a single ground station pass. Following a theoretical visibility of the satellite at $t_{\mathrm{b}}=0$ with a maximum range of about $3200 \mathrm{~km}$, a maximum elevation angle of $90 \mathrm{deg}$ and a minimum slant range of $700 \mathrm{~km}$ is achieved at $t=7.3$ mins. We have indicated three transmission scenarios in Fig. 1. Scenario I is a typical conventional operations scenario where the transmission starts early in the pass and ends late at the expense of a low data rate. Scenario III shows the other extreme where only a small interval around culmination is used for transmission, however at a significantly high data. Both scenarios I and III cover the same area, thus have the same total data volume. In contrast, scenario II expresses the main statement of this paper, which is that the achievable total data volume can be optimized by taking the minimum elevation angle into account as well. 


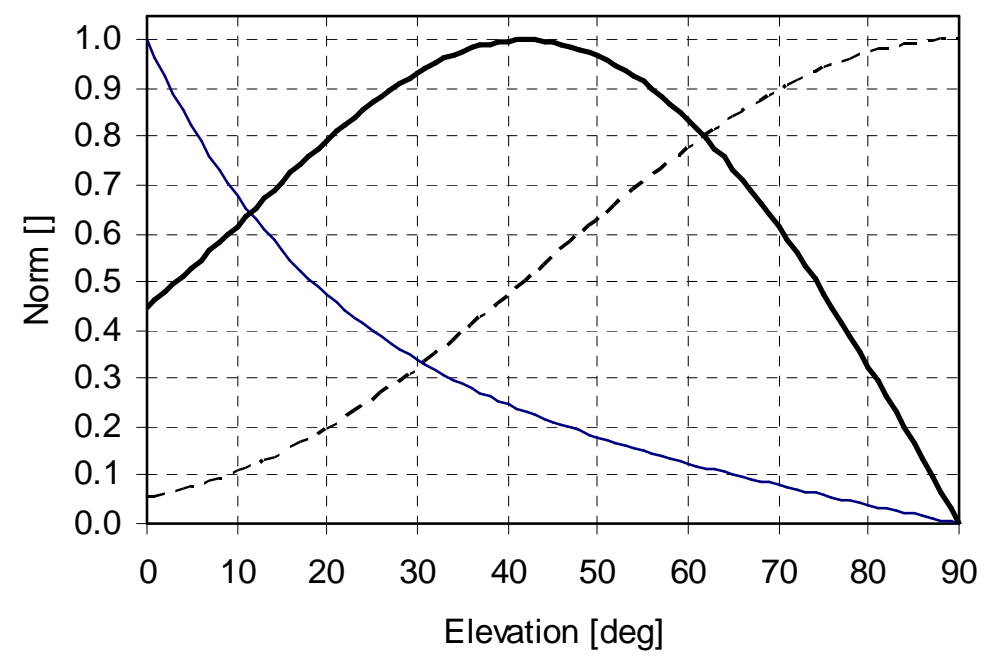

Figure 2 Normalized total data volume $D$ (bold line), normalized data rate $R$ (thin dashed line) and normalized transmission time $t_{\mathrm{t}}$ (thin solid line) as a function of the elevation. A sample satellite altitude of $700 \mathrm{~km}$ and a zenith pass are assumed.

With the expressions for data rate and pass duration as a function of the elevation, we arrive at the following equation for the total data volume $D$ per pass

$$
D(E)=R_{C} \frac{\lambda^{2} r^{3 / 2}\left(\pi-2 E-2 \arcsin \left(\cos E / f_{r}\right)\right.}{16 \pi^{2} \sqrt{G M_{\oplus}} R_{\oplus}^{2}\left(\sin E-\sqrt{f_{r}^{2}-\cos ^{2} E}\right)^{2}}
$$

where we apply for convenience the ratio $f_{\mathrm{r}}=r / R_{\oplus}$. The normalized total data volume is displayed in Fig. 2 as a function of the elevation $E$ together with its two constituents $R$ and transmission time $t_{\mathrm{t}}$. The data rate continuously increases with increasing elevation angle due to a decreasing range. At lower and in particular at higher elevation, this increase is less pronounced than at medium elevation angles. The transmission time continuously decreases with increasing elevation angle and vanishes at zenith. The product of the data rate and transmission time is the total transmitted data volume which shows a pronounced maximum at elevations between 40 and 45 deg for LEO satellites. This maximum elevation angle will be determined in the sequel.

Based on Eq. (5), we determine the derivative of $D$ as a function of the elevation $E$ which vanishes for an optimum value of the elevation $E_{\mathrm{opt}}$. This leads to

$$
0=\left(\sqrt{f_{r}^{2}-\cos ^{2} E_{o p t}}-\sin E_{\text {opt }}-\cos E_{\text {opt }}\left(\pi-2 E_{\text {opt }}-2 \arcsin \left(\cos E_{\text {opt }} / f_{r}\right)\right)\right)
$$

Eq. (7) is a transcendent function of the eccentricity. It is striking that Eq. (7) contains only a single parameter $f_{\mathrm{r}}$ which depends on the satellite altitude $h$ for a given Earth radius.

Although this work is focused on optimizing received data volume for single ground station contacts, in practice ground station operations must take into account preparation time $t_{\text {prep }}$, such as initialization and reconfiguration time, outages and other operational constraints. This can be accounted for by a relation for the mean data rate according to (Wertz \& Larson 1999).

$$
\bar{D}=R\left(F\left(t_{e}^{\max }-t_{b}^{\max }\right)-t_{\text {prep }}\right) / M,
$$

where $M$ is the margin needed for expected missed passes due to ground station downtime and $F$ is the fractional reduction in viewing time due to non-zenith passes over the ground station. In Eq. (2), $t_{t}^{\max }=t_{e}^{\max }-t_{b}^{\max }$ indicates the maximum visibility time for a zenith pass. 


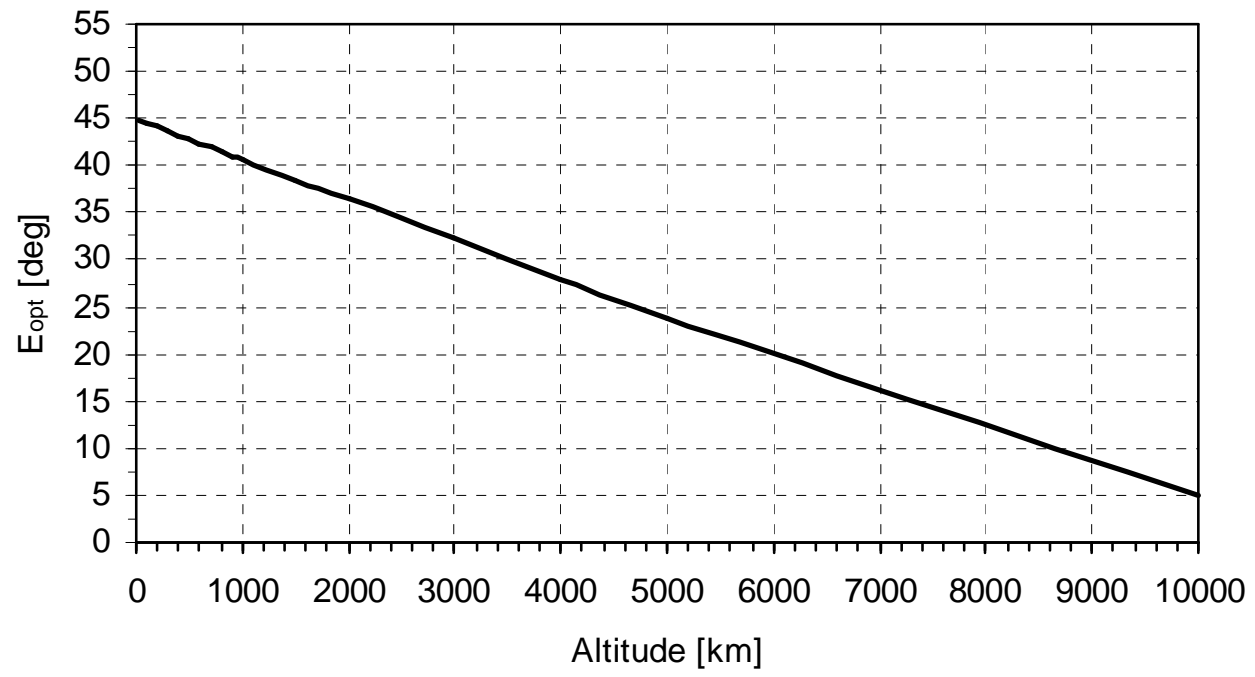

Figure 3 Optimum elevation for begin and end of data transmission as a function of orbital altitude of satellites in a circular orbit.

The root of Eq. (7) has been solved semi-analytically as a function of altitude and is displayed in Fig. 3. In the height regime below $10,000 \mathrm{~km}$, the optimum elevation to begin and end data transmission is decreasing with increasing altitude from an elevation angle of $45 \mathrm{deg}$ to a value of about 5 at an altitude of $10,000 \mathrm{~km}$. Above $10,000 \mathrm{~km}$ altitude the herein described method provides no advantage as compared to traditional schemes of satellite operations. Further considering that only few satellites are operated in the altitude regime between 1,000 and $10,000 \mathrm{~km}$, the herein described method is particularly suitable for LEO satellites.

A linear model of the optimum elevation angle $E_{\mathrm{opt}}$ as a function of altitude $h$ below $900 \mathrm{~km}$ is given by $E_{\text {opt }} \approx 45^{\circ}-0.00446 h[\mathrm{~km}]$.

By way of example, for a LEO satellite in a circular orbit at an altitude of $700 \mathrm{~km}$, the optimum elevation angle to start communication is $41.88 \mathrm{deg}$. This model provides an approximation of the optimum elevation angle with an error of less than $2^{\prime}$.

The benefit of high data rates at high elevations is not constant but depends on the altitude. This is illustrated in Fig. 4 as ratio of maximum to minimum data possible data rates as a function of altitude. Again, zenith passes are applied for illustration, where the minimum range is given as $h$ and the maximum range as $h \sqrt{1+2 R_{\oplus} / h}$. The conclusion that the optimum minimum elevation angle is much different from typical values applied in operations is also valid for non-zenith passes which are, however, much more complicated to treat.

\section{Ground Station Operations}

In the following, the above proposed approach is discussed for ground station operations. First, the context and needs of ground station operations is introduced followed by a description of a sample ground station operations system. Then, we discuss the opportunities and challenges of the proposed approach for ground station operations.

\section{A. Context and Needs}

Operating LEO missions is a complex task which requires involves interfaces, processes, tools, facilities and infrastructure and personal. The operations of ground stations is an important element within mission operations. Key requirements of ground station operations are

- Reliable communication with the space segment

- Real-time interface with mission control center

- Calibration of data and equipment

- Secure data archiving

- Maintenance of equipment and training of personnel. 


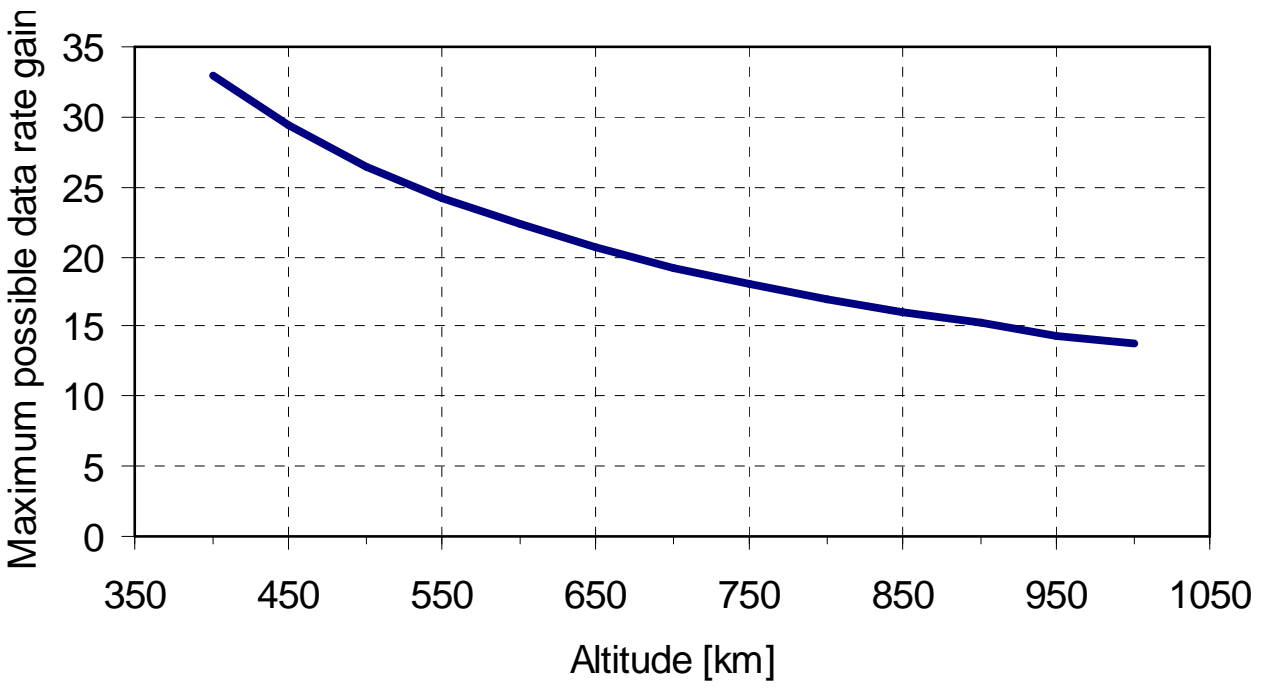

Figure 4 Ratio of maximum and minimum data rate possible for a zenith pass as a function of orbital altitude.

As ground station infrastructure and staff are costly, it is of interest to minimize ground station operations cost ${ }^{20}$ while being compliant to the mission requirements. A ground station complex typically comprises several antennas which have to support various satellite missions. As the allocation of support requests can be significant for a single ground complex, conflicting requests and a demand higher than the possible supply force ground station operators and space agencies towards standardization and cross-support ${ }^{21}$. A reduction of antenna time, if possible, is therefore of general interest to the operator.

From a satellite user perspective, performance and cost of ground station support are key parameters. Each hour of support requested from a ground station costs typically several thousand Euros, depending on the mission requirements and the ground station characteristics. Thus, minimal use of ground station time while satisfying the mission requirements is of interest for the user of satellite data.

\section{B. Ground Station Complex and Mission Support}

Ground station operations is an inherent part of mission operations. A sample network overview with emphasis to the ground stations and the mission operations system is shown for the GRACE mission in Fig. 5. The Grace mission $^{22}$ comprises two spacecraft flying in LEO which are separated in along-track direction by about $250 \mathrm{~km}$. The primary objective of the mission is to provide with unprecedented accuracy estimates of the global highresolution models of the Earth's gravity field. Apart from Launch and Early Operations (LEOP) and contingency support, two main ground station complexes are operationally supporting GRACE. The $15 \mathrm{~m} \mathrm{S-Band} \mathrm{antennas} \mathrm{in}$ Weilheim are used for spacecraft operations including telemetry and telecommand functions while the $7.2 \mathrm{~m} \mathrm{S-Band}$ antennas of Neustrelitz are only used for payload downlink.

Typical acquisition of the satellite signal commences at around $10 \mathrm{deg}$ elevation, depending on the mission and ground station configuration and the environment, such as elevation masks. Prior to the satellite pass itself, a preparation phase is required with activities such as pre-calibration and station and antenna configurations. Similarly, after the pass, a release time might be required with activities such as post-calibration and data archiving. 


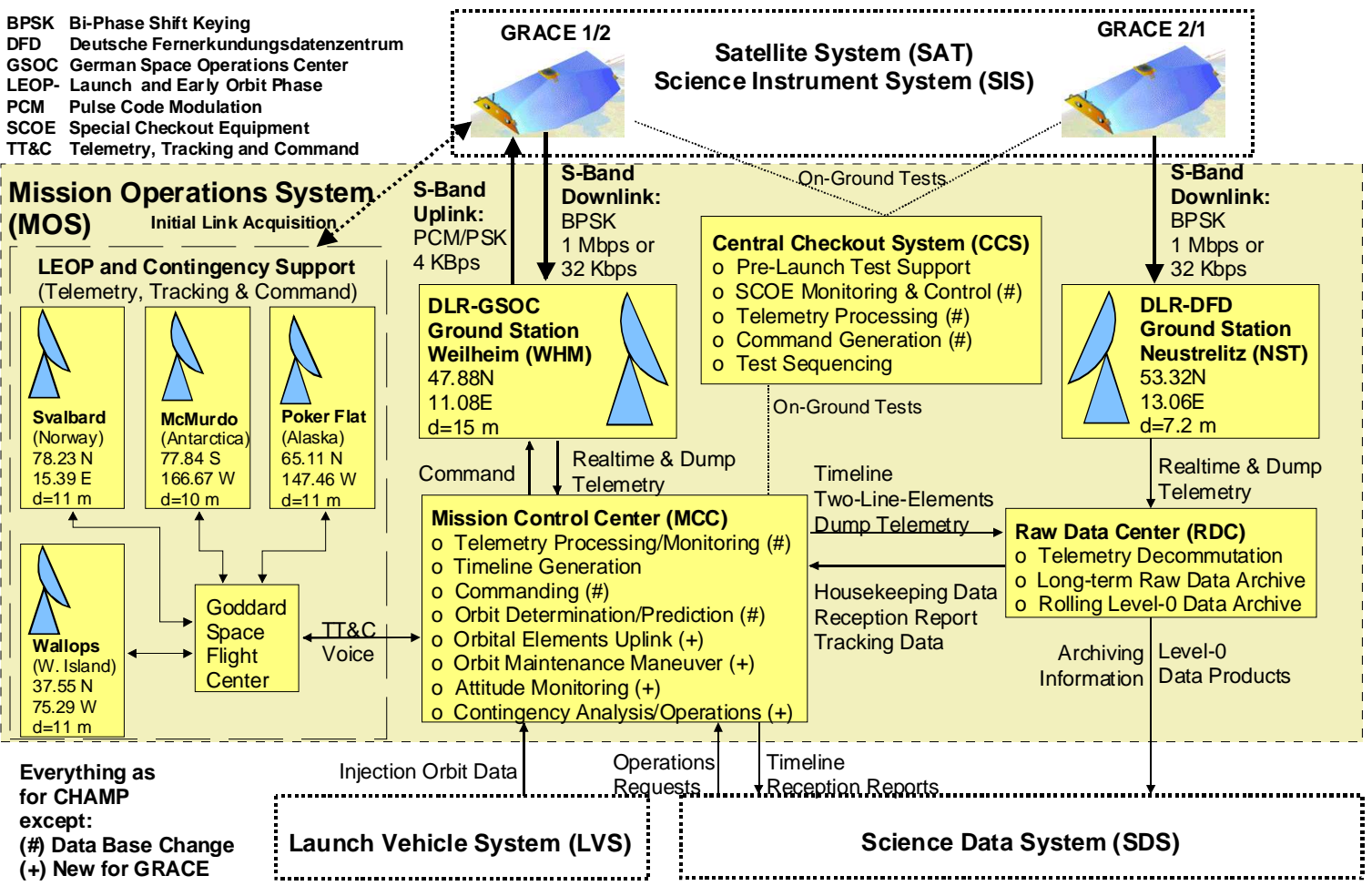

Figure 5 Sample mission operations system for the GRACE science mission ${ }^{23}$.

\section{Opportunities and Challenges}

The above presented approach suggests to limit communication time between a LEO satellite and a ground station to elevations higher than a certain and significant threshold. This will, as compared to traditional operations concepts, provide an increased total data volume per pass while limiting, at the same time, the necessary communication time to the spacecraft. A prerequisite to this approach is the technology required to apply an appropriate data rate for the downlink.

The approach has been worked out in the simplifying case of a LEO spacecraft in a circular orbit in a zenith pass over a ground station. For a spacecraft at an altitude of $700 \mathrm{~km}$, the regime of elevation angles for communication suggested in this paper ranges from 42 to $90 \mathrm{deg}$. While the total time of visibility of a single pass for an elevation threshold of 0 deg amounts to 14.0 mins, the communication time from 42 deg through 90 deg back to 42 deg is only 3.3 mins. Thus, a reduction of communication time of about $75 \%$ is achieved as compared to a traditional scenario. This reduction frees, in principle, ground station time which can be used to support e.g. other missions. Similar reductions are expected for non-zenith passes. This benefit will be even larger for satellites at lower altitude. The following provides a comprehensive summary of opportunities and challenges, which have been identified.

Opportunities:

- Time freed at ground stations

- Improved cross-support between different antennas (complexes)

- Higher data volumes

- Freed backup time in lower elevations regimes, if main operations scenario lead to problems

- Less losses and atmospheric considerations for the link

Challenges:

- Less communication time

- Could mean a increased risk of operations due to the reduced communication time

- Acquisition of signal at later times, thus a higher risk e.g. of not being able to track the spacecraft

- Less time to command, so could be especially interesting for $\mathrm{s} / \mathrm{c}$ with a high downlink data rate

Based on the complexity of mission and ground station operations, it is obvious that integrating the presented approach would have a significant impact on both concepts which has to be thoroughly studied. Based on the sample 
GRACE mission, introduced above, it is obvious that opportunities can, e.g., be realized in particular for stations dedicated to payload data downlink, while minimizing the risk of mission operations with different ground station complexes where conventional operations are performed.

\section{Summary and Conclusions}

While conventional satellite operations establish communication from low elevation angles onwards, it has been found that, assuming a fixed rate during actual transmission, a higher total data volume can be transmitted per pass when establishing communication only from an optimum elevation angle onwards. In the simplifying case of a circular LEO satellite orbiting at $700 \mathrm{~km}$ altitude in a zenith pass over the ground station, a maximum total data volume for the downlink is obtained when establishing communications from an optimum elevation angle of $41.88^{\circ}$ onwards. This angle is significantly different from typical elevation thresholds of $10^{\circ}$ used in normal satellite operations. It is remarkable that this optimum elevation threshold depends only on the ratio of the orbital radius to the radius of the Earth. The gain in total data volume which can be transmitted during one pass is about $40 \%$ for the considered case.

This result originates from the fact that at higher elevations, ranges between the satellite and the ground station are smaller than those close to the horizon, thus enabling higher data rates at a given onboard power for communications. Thus, the minimum elevation angle should not be considered an input parameter for the link analysis, but a result. This finding can significantly reduce the communication time needed for communications and operations to LEO satellites and there have consequences on optimizing the scheduling, and thus reducing cost or increasing capabilities of ground station complexes. Maximizing the total downlinked data volume per pass and optimizing the operational schedule comes, however, at the expense of a reduced communication time and a potentially enhanced risk of operations, unless proper counter actions are taken.

\section{References}

${ }^{1}$ Dietrich F.J., and Davies, R. S., “Communications Architecture”, Space Mission Analysis and Design, edited by J. R. Wertz and W. J. Larson, $3^{\text {rd }}$ ed., Kluver Academic Publishers, Dordrecht, 1999, pp. 533-570

${ }^{2}$ Wertz J. R., "Space Mission Geometry", Space Mission Analysis and Design, edited by J. R. Wertz and W. J. Larson, $3{ }^{\text {rd }}$ ed., Kluver Academic Publishers, Dordrecht, 1999, pp. 98-130

${ }^{3}$ European Corporation for Space Standardization (ECSS), Space Engineering - Radio Frequency and Modulation, ECSS-S50-05A 24 January 2003

${ }^{4}$ Cheung K., Lee C.H., Gearhart W.B., Vo T., Sindi S., Link-Capability Driven Network Planning and Operations, IEEEAC \#188, 2002 pp. 7-3281-7.3285

${ }^{5}$ Cowley W., Performance Comparisons for Adaptive LEO Satellite Links, Int. J. Satell. Commun. Networks, 2006, 24, pp. 229-239

${ }^{6}$ Miura A., and Tanaka M., Examination of the Data Rate Obtainable with low-gain non-tracking antenna applied to user terminal used for mobile communications and broadcast services provided by quasi-zenithal satellites, Int. J. Satell. Commun. Networks, 2005, 23, pp. 173-190

${ }^{7}$ Bryant G. H., Adimula I., Riva C., and Brussaard G., Rain attenuation statistics from rain cell diameters and height, Int. J. Satell. Commun. Networks, 2001, 19, pp. 263-283

${ }^{8}$ de Milliano, M., "Top-Level Design of the Communication System of the Delfi-Next Nano-Satellite", Master Thesis, Faculty of Aerospace Engineering, Delft University of Technology, Delft, The Netherlands, 2009.

${ }^{9}$ Matsuzawa, A., Abidi, A., Bogdan Staszewski, R., Craninckx, J., Dawson, J.L., Lee, T.C., Murmann, B., and Bang, S., "Digitally assisted analog and RF circuits: Potentials and issues", Solid-State Circuits Conference, A-SSCC '08. IEEE Asian Nov. 2008, pp. 485-485

${ }^{10}$ Bogdan Staszewski R., Muhammad K., Eliezer O., "Digital RF Processor (DRP) for Mobile Phones", Radio Frequency Integrated Circuits (RFIC) Symposium, IEEE, 2007, pp. 181-184

${ }^{11}$ Saeed, R., "Cognitive Radio and advanced spectrum management", Int. Conference on Communications, Computers and Applications, 2008. MIC-CCA 2008. Mosharaka, Aug. 2008, pp. xii

${ }^{12}$ Goldsmith, A., Jafar, S.A., Maric, I., and Srinivasa, S., "Breaking Spectrum Gridlock With Cognitive Radios: An Information Theoretic Perspective", Proceedings of the IEEE Vol. 97, No. 5, May 2009

${ }^{13}$ Cavdar, D., Yilmaz, H.B., Tugcu, T., Alagoz, F. , Compote., B., "Resource planning in cognitive radio networks", $6{ }^{\text {th }}$ International Symposium on Wireless Communication Systems, 2009. ISWCS 2009. 7-10 Sept. 2009

${ }^{14}$ Kitsunezuka, M., Hori, S., Maeda, T., "A Widely-Tunable, Reconfigurable CMOS Analog Baseband IC for SoftwareDefined Radio”, IEEE Journal of Solid-State Circuits, Vol. 44, No. 9, Sept. 2009, pp. 2496-2502

${ }^{15}$ Telli, A., Askar, M., "CMOS LNA design for LEO space S-band applications", Canadian Conference on Electrical and Computer Engineering, 2003. IEEE CCECE 2003, pp. 27-30 
${ }^{16}$ Fanucci, L., "Design of an ASIC for fast signal recognition and code acquisition in DS-SS-CDMA receivers", RF Syst. Div., Eur. Space Res. \& Technol. Centre, Noordwijk, Netherlands, Proceedings of the IEEE 4th International Symposium on Spread Spectrum Techniques and Applications, 1996, pp. 37-41

${ }^{17}$ Kuhn, W., Lay, N.E., Grigorian, E., Nobbe, D., Kuperman, I., Jeongmin Jeon, Kai Wong, Tugnawat, Y., Xin He, “A Microtransceiver for UHF Proximity Links Including Mars Surface-to-Orbit Applications", Proceedings of the IEEE, Oct. 2007, pp. 2019-2044

${ }^{18}$ de Milliano, M., Verhoeven, C.J.M., "Towards the Next Generation of Nanosatellite Communication Systems"; International Astronautical Congress, IAC-08.B2.1.3, Glasgow 2008

${ }^{19}$ Matinmikko, M., Mustonen, M., Sarvanko, H., Hoyhtya, M., Hekkala, A., Mammela, A., Katz, M., and Kiviranta, M., "A motivating overview of cognitive radio: Foundations, regulatory issues and key concepts", First International Workshop on Cognitive Radio and Advanced Spectrum Management, 2008, pp. 1.5

${ }^{20}$ Holdaway R., "The History and Continuing Quest to Reduce the Costs of Spacecraft Ground stations and Operations", Reducing the Cost of Spacecraft Ground Systems and Operations, edited by J. J. Miau J. J. and R. Holdaway, Kluver Academic Publishers, Dordrecht, 2000, pp. 1-8

${ }^{21}$ Pilgram M., "SLR Services - A Step to Standardization in Cross Support", Reducing the Cost of Spacecraft Ground Systems and Operations, edited by J. J. Miau J. J. and R. Holdaway, Kluver Academic Publishers, Dordrecht, 2000, pp. 17-24

${ }^{22}$ Tapley B.D., Bettadpur S., Watkins M. and C. Reigber, 2004, The Gravity Recovery and Climate Experiment: Mission overview and early results, Geophys. Res. Lett., 31, L09607, doi:10.1029/2004GL019920

${ }^{23}$ Kuch Th., Mühlbauer P.; "Raumfahrtmission GRACE", Deutsches Zentrum für Luft- und Raumfahrt (DLR) e.V., German Space Operations Center, 01 March 2002 